\title{
Toward Large-Scale Production of Oxidized Graphene
}

\author{
Talia Tene ${ }^{1}$, Gabriela Tubon Usca ${ }^{2,3}$, Marco Guevara ${ }^{2}{ }^{-0}$, Raul Molina ${ }^{4}$, Francesco Veltri ${ }^{3,5}$, \\ Melvin Arias ${ }^{3,6}$, Lorenzo S. Caputi ${ }^{3,5}$ and Cristian Vacacela Gomez ${ }^{3,7, *(1)}$ \\ 1 Department of Chemistry and Exact Sciences, Universidad Técnica Particular de Loja, Loja EC-110160, \\ Ecuador; tbtene@utpl.edu.ec \\ 2 Faculty of Science \& Mechanical Engineering, Escuela Superior Politécnica de Chimborazo, \\ Riobamba EC-060155, Ecuador; gabriela.tubon@espoch.edu.ec (G.T.U.); \\ marco.guevara@espoch.edu.ec (M.G.) \\ 3 UNICARIBE Research Center, University of Calabria, I-87036 Rende (CS), Italy; \\ francesco.veltri@unical.it (F.V.); melvin.arias@intec.edu.do (M.A.); lorenzo.caputi@fis.unical.it (L.S.C.) \\ 4 GraphenTech NL, Olympiaweg 28A, 3077AL Rotterdam, The Netherlands; rm@redecua.com \\ 5 Surface Nanoscience Group, Department of Physics, University of Calabria, Via P. Bucci, Cubo 33C, \\ I-87036 Rende, Italy \\ 6 Instituto Tecnológico de Santo Domingo, Area de Ciencias Básicas y Ambientales, Av. Los Próceres, \\ Santo Domingo 10602, Dominican Republic \\ 7 CompNano, Yachay Tech University, School of Physical Sciences and Nanotechnology, Urcuquí EC-100119, \\ Ecuador \\ * Correspondence: cvacacela@yachaytech.edu.ec
}

Received: 9 January 2020; Accepted: 1 February 2020; Published: 6 February 2020

\begin{abstract}
The oxidative exfoliation of graphite is a promising approach to the large-scale production of graphene. Conventional oxidation of graphite essentially facilitates the exfoliation process; however, the oxidation procedure releases toxic gases and requires extensive, time-consuming steps of washing and reduction to convert exfoliated graphene oxide (GO) into reduced graphene oxide (rGO). Although toxic gases can be controlled by modifying chemical reactions, filtration, dialysis, and extensive sonication are unfavorable for large-scale production. Here, we report a complete, scalable, and green synthesis of $\mathrm{GO}$, without $\mathrm{NaNO}_{3}$, followed by reduction with citric acid (CA). This approach eliminates the generation of toxic gases, simplifies the washing steps, and reduces the time required to prepare rGO. To validate the proposed method, we present spectroscopical and morphological studies, using energy-dispersive X-ray spectroscopy (EDS), UV-visible spectroscopy, infrared spectroscopy, Raman spectroscopy, scanning electron microscopy (SEM), and transmission electron microscopy (TEM). Thermal gravimetric analysis (TGA) is used to analyze the thermal properties of GO and rGO. This eco-friendly method proposes a complete guideline protocol toward large-scale production of oxidized graphene, with potential applications in supercapacitors, fuel cells, composites, batteries, and biosensors.
\end{abstract}

Keywords: graphene; oxidized graphene; citric acid; large-scale production

\section{Introduction}

Graphene, a two-dimensional (2D) single atomic layer of $\mathrm{sp}^{2}$ carbon atoms [1], has attracted intense research interest, owing to its high surface area, excellent thermal and electrical properties, extended charge carrier mobility, high elastic behavior, and optical tunability [1-5]. Transferable single-layer graphene was first obtained by mechanical exfoliation [6] of bulk graphite and by chemical vapor deposition [7]. Although these routes produce high-quality graphene, they can be less effective for large-scale manufacturing. The major obstacle to achieving single-layer or few-layer 
graphene is the interlayer van der Waals interaction [8]. Keeping this consideration in mind, the most common approaches to the large-scale synthesis of graphene rely on liquid-phase exfoliation $[9,10]$ or oxidation-reduction of graphite [11,12]. The key differences between these methods are the yield and the defect content of their products. Defect-free graphene is mostly preferred for electronic applications [13], whereas in some other cases, defects and imperfections in graphene are less relevant and desirable [14-16]. Some achievements have improved liquid-exfoliation processes [17-19] to obtain large quantities of graphene. However, the obtained graphene mainly has a hydrophobic feature and a small lateral size. Thus, the oxidation-reduction of graphite is a more practical method for bulk-scale graphene materials in immediate applications-e.g., to produce colloidal suspensions for versatile processing of thin films and composites [20,21].

Typically, the oxidation-reduction of graphite uses strong oxidizing agents to yield graphene oxide (GO), an insulating carbon material [22]. To date, the basic structure of GO remains ambiguous due to the vast number of functional groups and the entailed isomeric possibilities. It is well-known that, depending on the degree of oxidation, the aromatic lattice of graphene exhibits different oxygen functional groups, such as hydroxyl, epoxy, carboxyl, and carbonyl groups [22-24], which increase the interlayer spacing from $0.33 \mathrm{~nm}$ (graphite) to $0.87 \mathrm{~nm}$ (GO) [25]. At a low degree of oxidation, hydroxyl groups can chemically turn into epoxide groups and vice versa, allowing for the use of GO for specific technological applications [24]. In addition, the GO structure reveals a mixed $\mathrm{sp}^{2}-\mathrm{sp}^{3}$ hybridization as a direct consequence of the degree of oxidation acquired during the oxidation process [26] (see, e.g., Brodie [27], Staudenmaier [28], Hummer [29], Marcano [30], and Chen [31]). Hummer et al. reported the method most used nowadays: the oxidation of graphite is realized by treatment with $\mathrm{KMnO}_{4}$ and $\mathrm{NaNO}_{3}$ in concentrated $\mathrm{H}_{2} \mathrm{SO}_{4}$. This procedure involves the generation of toxic gases, such as $\mathrm{NO}_{2}$ and $\mathrm{N}_{2} \mathrm{O}_{4}$, and the residual $\mathrm{Na}^{+}$and $\mathrm{NO}_{3}{ }^{-}$ions are difficult to remove during the processes of synthesizing and purifying GO [29-31]. While the induced oxygen functional groups increase the distance between graphitic layers (weakening the van der Waals forces and facilitating the preparation of GO), the removal of oxidizing agents and other impurities from GO is obtained through conventional methods such as filtration, dialysis, or sonication, making the Hummer method unsuitable for scaling at an industrial level.

To circumvent these environmental and technical issues, Chen et al. [31] have improved the Hummer method by excluding $\mathrm{NaNO}_{3}$. The authors successfully demonstrated that this modification does not decrease the yield of the product, but reduces toxic gas evolution and makes the disposal of wastewater more straightforward due to the absence of $\mathrm{Na}^{+}$and $\mathrm{NO}_{3}{ }^{-}$ions [31]. Nevertheless, the obtained graphite oxide needs to be filtered and purified by dialysis for one week to remove metal ions. Additionally, the resulting graphite oxide needs to be diluted in $1.2 \mathrm{~L}$ of water, stirred overnight, and sonicated for $30 \mathrm{~min}$ to give, finally, GO powder. For large-scale applications of graphene, the filtration and dialysis are a bottleneck. These steps are time-consuming, since graphite oxide particles (especially exfoliated ones) rapidly block the filter and membrane pores. Also, the reduction process adds yet another step to the synthesis procedure, prolonging the overall production time [32]. Hence, there is a great challenge to develop a green, low-cost, and efficient reduction method.

To support this direction, Abdolhosseinzadeh et al. [33] reported a sonication-assisted oxidation method for large-scale production of GO followed by the reduction of GO with ascorbic acid before washing steps, which relatively decreased the production time of reduced graphene oxide (rGO). This method proposes the preparation of GO by the Hummer method, using a stirring-sonication process of $5 \mathrm{~min} 12$ times, and an extra ultrasonication for $2 \mathrm{~h}$ prior to reduction. After reduction, the obtained material is sonicated for $1 \mathrm{~h}$ and filtrated to obtain rGO powder. Nevertheless, the procedures involving extended sonication have limited scalability using an extensive sonication time, which may induce basal/edge defects, a small lateral size, and high-cost production [34]. Recently, Garino et al. [35] reported a microwave-assisted fast synthesis of rGO co-doped with manganese and nitrogen through a green approach. The co-doping with nitrogen and manganese occurs while reducing GO. 
To the best of our knowledge, a reliable method with the capability to supply the large demand for pristine GO and (undoped) rGO by using a green approach, a short-sonication time, and viable washing steps is still lacking. In this communication, such a method is presented, considering the environmental problem and technical limitations. We successfully demonstrate, through several spectroscopy and morphological studies, an eco-friendly and complete guideline protocol for large-scale production of GO by excluding $\mathrm{NaNO}_{3}$ [31] and adding citric acid (CA) after a simple washing process. The advantages of this method, with its simple protocol, preserved yield, and lack of extensive sonication and toxic gas evolution during preparation of GO, make it attractive for preparing rGO on a large scale, with promising applications in composites, energy storage, and reinforcement, where a large quantity of oxidized graphene is needed [36].

\section{Materials and Methods}

Graphite powder $(<150 \mu \mathrm{m}, 99.99 \%)$, sulfuric acid $\left(\mathrm{H}_{2} \mathrm{SO}_{4}, \mathrm{ACS}\right.$ reagent, 95.0-98.0\%), potassium permanganate $\left(\mathrm{KMnO}_{4}, \mathrm{ACS}\right.$ reagent, $\left.\geq 99.0 \%\right)$, hydrochloric acid $(\mathrm{HCl}, \mathrm{ACS}$ reagent, 37\%), and citric acid $\left(\mathrm{C}_{6} \mathrm{H}_{8} \mathrm{O}_{7}\right.$, ACS reagent, $\left.\geq 99.5 \%\right)$ were obtained from Sigma Aldrich. All chemicals were used as received, without further purification. Figure 1 shows the schematic route of the production line for GO and rGO.

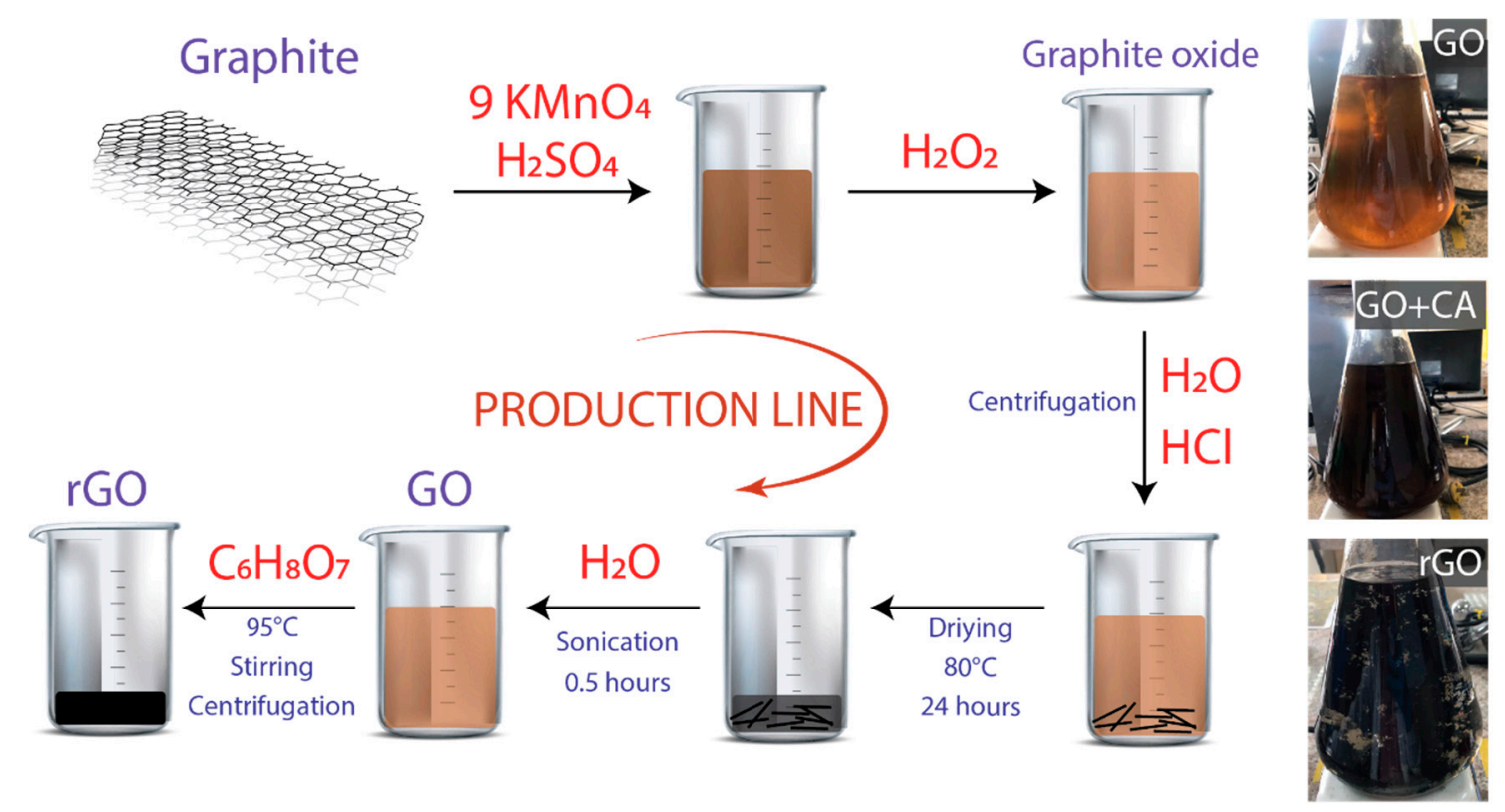

Figure 1. Schematic representation of the procedure. The starting material is graphite powder. Inset: optical images of graphene oxide (GO), GO plus citric acid (CA), and reduced graphene oxide (rGO).

\subsection{Synthesis of $\mathrm{GO}$}

It is important to stress once more that the synthesis of GO is based on the approach of Ref. [31]. However, some obvious modifications were developed to make the process as simple as possible. In the typical experiment, $3.0 \mathrm{~g}$ of graphite powder was added to $70 \mathrm{~mL}$ of concentrated $\mathrm{H}_{2} \mathrm{SO}_{4}$ while stirring in an ice-water bath. Then, $9.0 \mathrm{~g}$ of $\mathrm{KMnO}_{4}$ were gradually added, maintaining the temperature of the suspension to be lower than $20{ }^{\circ} \mathrm{C}$. The resulting mixture was transferred to a $50{ }^{\circ} \mathrm{C}$ oil bath and vigorously agitated for about $0.5 \mathrm{~h}$. Distilled water $(150 \mathrm{~mL})$ was added, and the solution was stirred for $20 \mathrm{~min}$ at $\sim 90^{\circ} \mathrm{C}$. Additionally, $500 \mathrm{~mL}$ distilled water was added, followed by a slow addition of $15 \mathrm{~mL}$ of hydrogen peroxide $\left(\mathrm{H}_{2} \mathrm{O}_{2}, 30 \%\right.$, Merk) and stirred up to turn the color of the solution from dark brown to yellowish. The resulting graphite oxide suspension was washed with 1:10 $\mathrm{HCl}$ solution and distilled water several times, each for $10 \mathrm{~min}$ by means of centrifugation at $3000 \mathrm{rpm}$ until the $\mathrm{pH}$ was at $\sim 6$. The precipitated material was dried at $80{ }^{\circ} \mathrm{C}$ for $24 \mathrm{~h}$. 


\subsection{Synthesis of $r G O$}

Initially, $50 \mathrm{mg}$ of resulting solid was dispersed in $500 \mathrm{~mL}$ of distilled water by sonication for $0.5 \mathrm{~h}$, employing an ultrasonic bath (Branson 2510 Ultrasonic Cleaner, Framingham, MA, USA) in continuous operation. Bath sonication is preferred to tip sonication because the direct positioning of the tip in the medium results in strong material fragmentation [37]. The obtained suspension was centrifugated at $1000 \mathrm{rpm}$ for $0.5 \mathrm{~h}$ and divided into two equal parts: one to obtain a homogenous GO suspension (Figure 1, optical images), and the other was further processed for preparing rGO. Under vigorous agitation, $250 \mathrm{mg}$ CA was slowly added in the remaining suspension. Different reduction times were tested (from 0.5 to $3 \mathrm{~h}$ ), setting the reduction temperature at $95^{\circ} \mathrm{C}$. To remove excess CA, the resultant black precipitates were washed with distilled water by centrifugation at $3000 \mathrm{rpm}$ for $0.5 \mathrm{~h}$. Finally, the precipitated material was dried at $80^{\circ} \mathrm{C}$ overnight to obtain rGO powder.

\subsection{Characterization}

The characteristic absorption spectra of GO and rGO were recorded using UV-vis spectroscopy (Thermo Scientific, Evolution 220, Waltham, MA, USA). Infrared spectra were collected using a Fourier transform infrared spectrometer Jasco FT/IR-4000 (Easton, MD, USA). The thermal stability of GO and rGO was investigated using thermogravimetric analysis (TGA, PerkinElmer simultaneous thermal analyzer, STA 6000, Waltham, MA, USA). The structure and surface morphology of the obtained materials were taken out on a transmission electron microscope (TEM, JEM 1400 Plus, JEOL, Musashino, Akishima, Tokyo, Japan) operating at $80 \mathrm{kV}$, and a scanning electron microscope (SEM, JSM-IT100 InTouchScope, JEOL, Musashino, Akishima, Tokyo, Japan) equipped with a JEOL-made dispersive $\mathrm{X}$-ray spectrometer (EDS) with the accelerating voltage of $15 \mathrm{kV}$. Raman spectra were obtained using a Jasco NRS-500 (Easton, MD, USA) spectrometer with a $532 \mathrm{~nm}$ laser wavelength $(0.3 \mathrm{~mW}, 100 \mathrm{X}$ objective). SEM samples were prepared by drop casting on aluminum substrates and dried at $80{ }^{\circ} \mathrm{C}$ for $2 \mathrm{~h}$. Similarly, TEM and Raman samples were prepared by drop casting onto formvar-coated copper grids and glass substrates, respectively.

\section{Results and Discussion}

The main intent of this work is to propose a general eco-friendly approach to large-scale production of oxidized graphene. In this sense, we have selected CA because it is a green and low-cost alternative for conventional reducing agents of GO [38] (e.g., hydrazine hydrate, hydroquinone, sodium borohydride, and hydrogen sulfide), which can acceptably reduce the obtained GO as discussed in the following. Furthermore, CA together with hydrazine hydrate [39] or alkali hydroxide [40] has been used to prepare reduced graphene oxide or graphene oxide quantum dots.

After treatment with CA, the yellowish GO solution turned black, and a stable dispersion of rGO flakes could be detected by the naked eye, as displayed in Figure 1 (optical images). The optical change of GO (yellowish suspension) to $\mathrm{rGO}$ (black suspension) strongly confirms the reduction of oxygen functional groups (Figure $\mathrm{S} 1$ ). GO and rGO flakes are easy to precipitate as time increases. However, the stability of the GO suspension was higher than that of the rGO suspension within $72 \mathrm{~h}$. In general, graphene tends to aggregate and precipitate in aqueous media due to its hydrophobicity and the strong $\pi-\pi$ interactions between graphene layers. Therefore, the observed sedimentation supports the formation of rGO [41].

In order to investigate the elemental composition of GO and $\mathrm{rGO}$, we performed EDS measurements, controlling the reduction temperature and testing different reduction times (Figure 2a). As the EDS technique depends on the bombarded region, the investigated area was large enough to ensure the reliability of the results. As the interaction time increased between GO and CA, the reduction effectivity increased from 0.5 to $1.5 \mathrm{~h}$; however, a slight oxidation was observed after $2 \mathrm{~h}$. This result could be attributed to a partial oxygen functionalization from water molecules because the reduction was carried out at $95^{\circ} \mathrm{C}$. In particular, the observed reduction of oxygen element was 
about $7 \%$ at $0.5 \mathrm{~h}$ and $13 \%$ at $1.5 \mathrm{~h}$. We focused on the reduction at $0.5 \mathrm{~h}$ to optimize the production process, avoid a probable instable reduction, and preserve a good dispersibility in water. The latter is an important feature that may be exploited in antibacterial and cytotoxic applications [42]. Figure 2b-d (and Figure S2) reveal that pristine graphite, GO, and rGO are mainly composed of carbon and oxygen elements in the accelerating-voltage window from 0 to $5 \mathrm{keV}$.
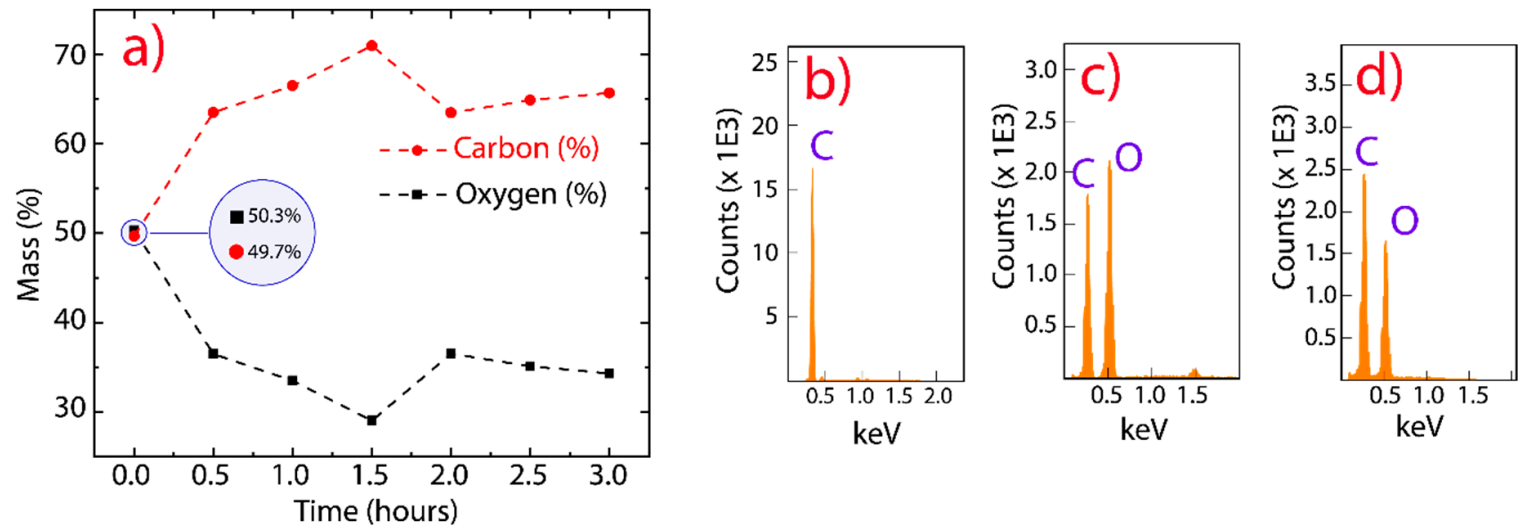

Figure 2. EDS analysis. (a) Elemental compositions (\%) as a function of the reduction time by using citric acid. (b-d) EDS spectra of graphite, GO, and rGO, respectively.

The reduction of GO into rGO was further confirmed by UV-vis absorption spectroscopy and the observed results are depicted in Figure 3a (and Figure S3). GO exhibits two absorption peaks (black line) at $233 \mathrm{~nm}$, and a shoulder peak at $304 \mathrm{~nm}$, which are attributed to the $\pi-\pi^{*}$ transitions of $\mathrm{C}-\mathrm{C}$, and $\mathrm{n}-\pi^{*}$ transitions of $\mathrm{C}=\mathrm{O}$ bonds, respectively. The absorption peak observed at $233 \mathrm{~nm}$ of GO has been red shifted to $263 \mathrm{~nm}$ for rGO (red line), suggesting that the electronic conjugation of the aromatic structure might be restored. The overall features of these spectra and their absorption peaks are similar to those of $\mathrm{GO}$ and $\mathrm{rGO}$ reported in the literature $[30,31,43]$. Most importantly, the rGO spectra, considering different reduction times, are featureless in the visible region, as expected for graphene (Figure S3).
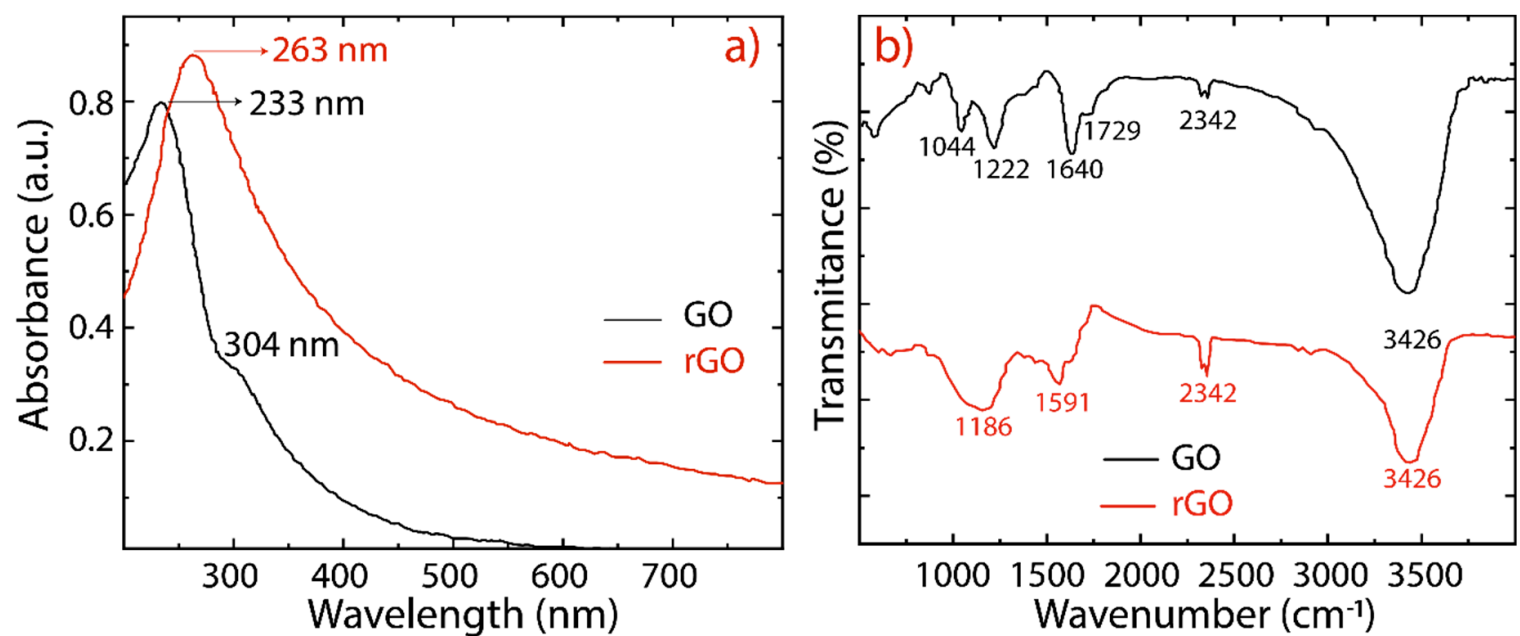

Figure 3. (a) UV-visible spectra recorded in aqueous solutions at $0.1 \mathrm{mg} / \mathrm{mL}$ of GO (black line) and rGO (red line). (b) Fourier transform infrared spectra of GO (black line) and rGO (red line).

The oxygen-containing functional groups are confirmed in the FTIR spectra given in Figure 3b. As is well-known, the hydroxyl and epoxide groups attached to the basal plane of the graphene and carboxyl and carbonyl groups located at the edges are the dominant functional groups [24]. The 
following characteristic functional groups are detected: $\mathrm{C}-\mathrm{O}-\mathrm{C}\left(1044 \mathrm{~cm}^{-1}\right), \mathrm{C}-\mathrm{O}\left(1222 \mathrm{~cm}^{-1}\right), \mathrm{C}=\mathrm{C}$ $\left(1644 \mathrm{~cm}^{-1}\right)$, and $\mathrm{C}=\mathrm{O}\left(1729 \mathrm{~cm}^{-1}\right)$. The broad peak observed at $3426 \mathrm{~cm}^{-1}$ is due to the hydroxyl groups $(\mathrm{O}-\mathrm{H})$ and adsorbed water molecules between $\mathrm{GO}$ sheets. This hydrophilic feature provides GO sheets with a good dispersibility in water. After reduction, the prominent peaks in the GO spectrum result are significantly attenuated and weakened in the rGO spectrum, confirming the removal of oxygen functional groups. The band observed at $2360 \mathrm{~cm}^{-1}$ in GO and $\mathrm{rGO}$ is assigned to the $\mathrm{CO}_{2}$ stretching vibration [44].

Figure 4 presents the TGA curves of GO and rGO. In GO, the minimal weight loss ( $~ 5 \%)$ before $100{ }^{\circ} \mathrm{C}$ is attributed to the loss of physisorbed water molecules [44]. The significant weight loss $(\sim 25 \%)$ in the range of $200-250{ }^{\circ} \mathrm{C}$ and weaker mass loss $(\sim 10 \%)$ in the range of $250-600{ }^{\circ} \mathrm{C}$ are attributed to the pyrolysis of less stable $\left(\mathrm{CO}, \mathrm{CO}_{2}\right.$, and $\left.\mathrm{H}_{2} \mathrm{O}\right)$ and more stable oxygenated functional groups, respectively [45]. On the other hand, rGO reveals a higher thermal stability compared to GO due to the graphitization and promoted van der Waals forces between the layers through the removal of oxygen functional groups [44]. TGA curves of both GO and rGO reflect a similar trend, suggesting their close contents of oxygenated groups, as the FTIR data indicated.

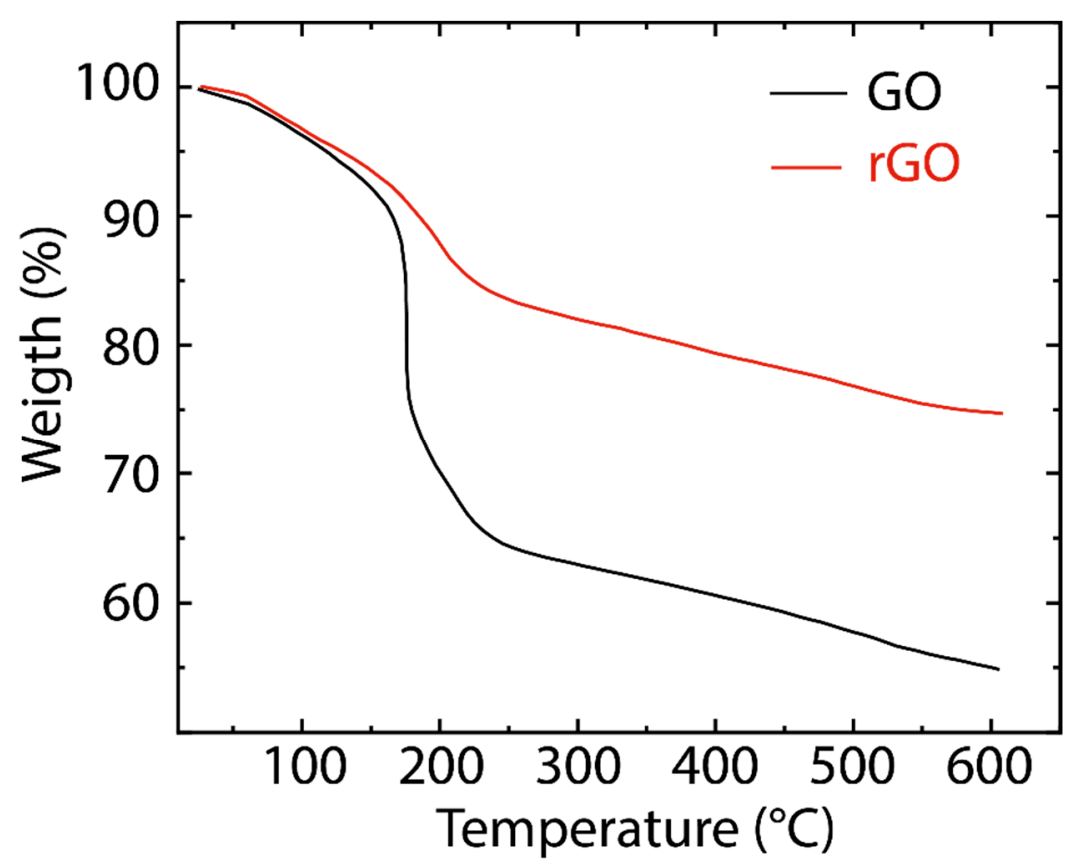

Figure 4. Thermogravimetric analysis (TGA) of GO (black line) and rGO (red line).

Raman spectroscopy was used to examine structural changes during the oxidation and reduction processes. The Raman spectra of GO and rGO are reported in Figure 5. As is typical for oxidized graphene, two prominent peaks are detected: i.e., the D peak at $\sim 1345 \mathrm{~cm}^{-1}$ and the $G$ peak at $\sim 1597 \mathrm{~cm}^{-1}$. Other less intense peaks also have been detected: the $2 \mathrm{D}$ peak at $\sim 2700 \mathrm{~cm}^{-1}$ and the $D+G$ peak at $\sim 2920 \mathrm{~cm}^{-1}$. The $D$ and $D+G$ (the combination of $D$ and $G$ bands) peaks are ascribed to structural imperfections of GO and rGO (vacancies, heptagon/pentagon rings, the edge effect [46-48]) while the $G$ peak is related to the in-plane bond-stretching motion of $\mathrm{sp}^{2}$-hybridized carbon atoms in the graphene/graphite lattice. On the other hand, the 2D peak is regularly used to estimate the number of layers in obtained graphene. 


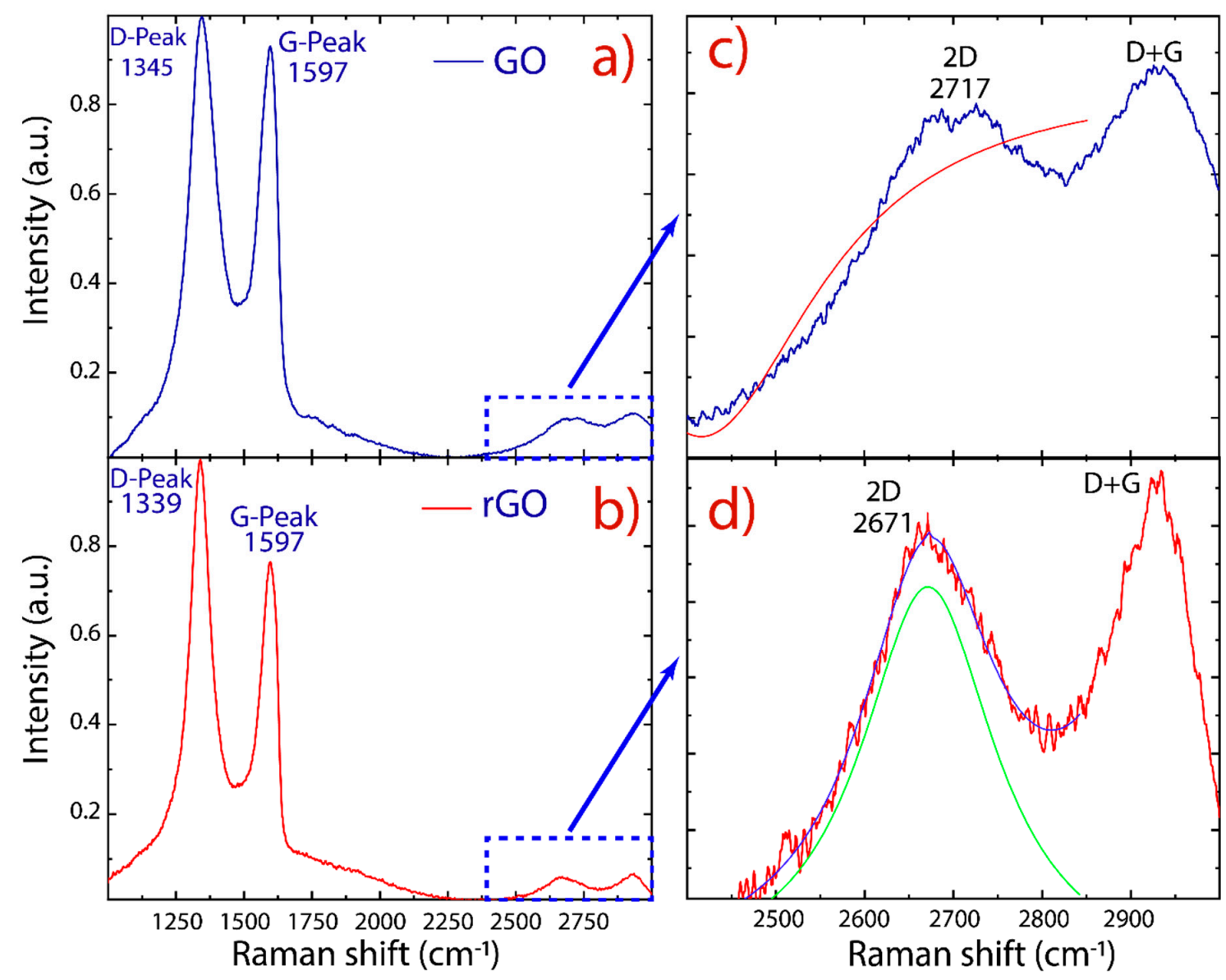

Figure 5. Raman spectra recorded using $532 \mathrm{~nm}$ laser excitations. (a) GO and (b) rGO. (c,d) Fitting of the $2 \mathrm{D}$ peaks with Lorentzian functions. The intensity was normalized by the $\mathrm{D}$ peak.

The peak ratio between the intensity of $\mathrm{D}$ and $\mathrm{G}$ peaks $\left(I_{D} / I_{G}\right)$ is a common index for the density of defects on GO and rGO. As shown in earlier studies [48-50], it was found that the intensity ratio of $\mathrm{rGO}(\sim 1.29)$ is larger than that of GO ( 1.09), indicating that the size of the graphene-like domains is diminished after exposure to reduction and sonication, but they are more numerous in number [48]. Interestingly enough, the $\mathrm{D}$ and $\mathrm{G}$ peaks of $\mathrm{rGO}$ are more separated from each other and are distinctly different from GO, where the D and G peaks are broad and overlap: i.e., the increased intensity of the $D$ peak to $G$ peak is due to the elimination of defects [33], suggesting that rGO did not undergo severe structural disruption compared to GO.

Unlike the 2D peak of monolayer graphene, which has high intensity, the 2D peak of GO and rGO is complex in shape and evolves with the number of layers [46]. The presence of an intense D peak and a weak 2D peak is a general feature of graphene produced by oxidation-reduction-based methods. However, the intensity of the 2D peak also depends on the excitation laser frequency, and therefore cannot be solely relied upon [51]. In this context, we used the full width at half maximum (FWHM) to evaluate the 2D peak. By fitting the 2D peak with Lorentzian functions, the 2D peak $\left(\sim 2671 \mathrm{~cm}^{-1}\right)$ of rGO is shifted with respect to the 2D peak $\left(\sim 2717 \mathrm{~cm}^{-1}\right)$ of GO. The latter was impossible to be fitted, and the maximum intensity value was removed from the data (Figure $5 \mathrm{c}$ ). The respective FWHM of rGO was found to be $183.6 \pm 7.0 \mathrm{~cm}^{-1}$. Furthermore, the 2D peak became more symmetrical in rGO, indicating the presence of few-layer-reduced graphene oxide.

SEM and TEM micrographs of GO and rGO are shown in Figure 6. The observed morphology of GO (Figure 6a) consists of randomly aggregated, transparent, and flake-like sheets with wrinkles and folds on the surface of GO, as well as face-to-face stacking of sheets. The surface morphology of rGO (Figure $6 \mathrm{~b}$ ) shows a significant difference compared to GO. A porous surface (after reduction 
and sonication) is discovered, and rGO shows a highly distorted surface that can prevent face-to-face stacking of the graphene layers by the formation of mesopores and macropores. The semitransparent layers in GO and rGO seem to be free of impurities, which can be interpreted as an important result to prove that extensive or time-consuming washing processes (e.g., filtration, dialysis, ultrasonication, sonication) can be evaded.
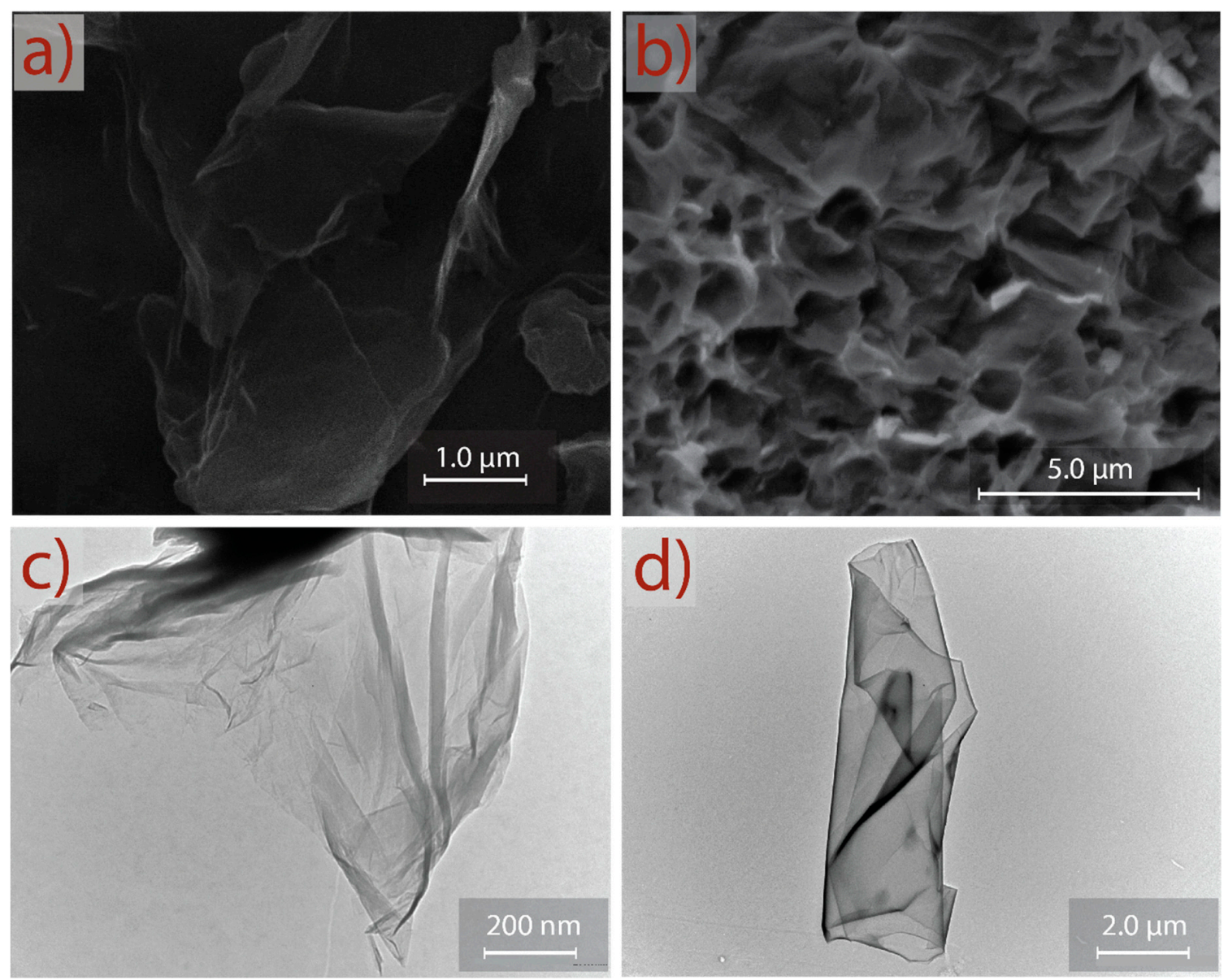

Figure 6. SEM morphology of (a) GO and (b) rGO. TEM images of (c) GO and (d) rGO.

This asseveration is supported by TEM analysis. Figure $6 \mathrm{c}$ shows a representative TEM image of GO. A transparent and thin nanosheet with some wrinkles and folds on the surface and edges is observed for GO. At first approximation, the GO nanosheet analyzed in this work appears to be very similar to the GO samples prepared by conventional oxidation-reduction-based methods; however, a clear difference is observed-i.e., the GO prepared by commonly used methods are mostly folded or have a folded edge due to a strong oxidation produced in the presence of $\mathrm{NaNO}_{3}[29,30]$. Figure $6 \mathrm{~d}$ presents a well-defined rGO nanosheet obtained after reduction, sonication, and washing. The observed regular surface suggests that the proposed method does not induce critical basal-plane damage during oxidation or reduction processes. These results confirm an eco-friendly and complete guideline protocol that can be implemented for the large-scale production of oxidized graphene through a reliable approach, short-sonication time, and simple washing steps.

\section{Conclusions}

In summary, we have established a complete and green protocol for the preparation of GO and rGO by excluding $\mathrm{NaNO}_{3}$ in the oxidation and adding CA in the reduction, which demonstrated to be an effective and inexpensive reducing agent for GO. EDS measurements showed a reduction 
effectivity from 0.5 to $1.5 \mathrm{~h}$, while GO is in contact with CA under vigorous agitation. UV-visible spectra confirmed the reduction of GO into rGO. FTIR analysis demonstrated the presence of oxygen functional groups, which decreased in intensity after reduction. The thermal stability of rGO was found to be higher compared to GO. Raman spectra showed a high intensity of the D peak in GO, indicating the presence of basal/edge defects; however, the characteristic 2D peak of graphene arose in rGO. SEM measurements showed that the semitransparent layers were relatively free of impurities. TEM analysis presented similar features. However, rGO exhibited a more regular, transparent, and thin nanosheet. The proposed method prevents toxic gas evolution, prolonged sonication, and complicated washing steps, making it effective at preparing oxidized graphene on a large scale.

Supplementary Materials: The following are available online at http://www.mdpi.com/2079-4991/10/2/279/s1, Figure S1. Optical images of rGO (black) suspensions. To obtain suspensions from rGO powder, the reduction of GO with CA was carried out considering three different times $(0.5,1.0,1.5 \mathrm{~h})$ with the procedure outlined in the main text. Then, $10 \mathrm{mg}$ of rGO powder were added into $10 \mathrm{~mL}$ distilled water and sonicated for $30 \mathrm{~min}$. The obtained suspensions were centrifugated for $30 \mathrm{~min}$ at $3000 \mathrm{rpm}$. Figure S2. EDS spectra of GO and rGO considering different reduction times $(0.5 \mathrm{~h}, 1.0 \mathrm{~h}$ and $1.5 \mathrm{~h})$. The carbon and oxygen elements are predominant in the range from 0 to $5 \mathrm{keV}$. Figure S3. UV-visible spectra recorded in aqueous solutions at $0.1 \mathrm{mg} / \mathrm{mL}$ of (a) GO and $(b-d)$ rGO considering different reduction times $(0.5 \mathrm{~h}, 1.0 \mathrm{~h}$ and $1.5 \mathrm{~h})$. The intensity was normalized by the predominant peak.

Author Contributions: R.M., C.V.G., and L.S.C. supervised the work. G.T.U. prepared the GO and rGO samples. M.G., and G.T.U. performed the EDS analysis and SEM observation. F.V., and L.S.C. conducted Raman and TEM measurements. T.T., G.T.U., M.G., and M.A. helped with the UV-vis, TGA, and IR validations. All authors contributed to the scientific discussion and manuscript preparation. T.T. and C.V.G. wrote the manuscript. All authors have read and agreed to the published version of the manuscript.

Funding: This research received no external funding.

Acknowledgments: D. Colcha, J. Pilamala, P. Angamarca, and A. Nuñez, undergraduate students of Escuela Superior Politécnica de Chimborazo, as well A. Valarezo and O. Salguero, undergraduate students of Yachay Tech University, participated in this project. T.T. and C.V.G. wish to thank Escuela Superior Politécnica de Chimborazo for hospitality during the completion of this work. The authors are very thankful to $\mathrm{H}$. Brito and F. Arias from the Faculty of Sciences-ESPOCH as well S. Briceño and J. Chacón-Torres from the School of Physical Sciences and Nanotechnology-Yachay Tech, for the facilities provided in the UV-vis characterization and stimulating discussions.

Conflicts of Interest: The authors declare no conflicts of interest.

\section{References}

1. Neto, A.C.; Guinea, F.; Peres, N.M.; Novoselov, K.S.; Geim, A.K. The electronic properties of graphene. Rev. Mod. Phys. 2009, 81, 109. [CrossRef]

2. Coello-Fiallos, D.; Tene, T.; Guayllas, J.L.; Haro, D.; Haro, A.; Gomez, C.V. DFT comparison of structural and electronic properties of graphene and germanene: Monolayer and bilayer systems. Mater. Today Proc. 2017, 4, 6835-6841. [CrossRef]

3. Sindona, A.; Pisarra, M.; Vacacela Gomez, C.; Riccardi, P.; Falcone, G.; Bellucci, S. Calibration of the fine-structure constant of graphene by time-dependent density-functional theory. Phys. Rev. B 2017, 96, 201408. [CrossRef]

4. Villamagua, L.; Carini, M.; Stashans, A.; Vacacela Gomez, C. Band gap engineering of graphene through quantum confinement and edge distortions. Ric. Mat. 2016, 65, 579-584. [CrossRef]

5. Vacacela Gomez, C.; Pisarra, M.; Gravina, M.; Sindona, A. Tunable plasmons in regular planar arrays of graphene nanoribbons with armchair and zigzag-shaped edges. Beilstein J. Nanotechnol. 2017, 8, $172-182$. [CrossRef]

6. Geim, A.K. Graphene prehistory. Phys. Scr. 2012, 146, 014003. [CrossRef]

7. Reina, A.; Jia, X.; Ho, J.; Nezich, D.; Son, H.; Bulovic, V.; Kong, J. Large area, few-layer graphene films on arbitrary substrates by chemical vapor deposition. Nano Lett. 2008, 9, 30-35. [CrossRef]

8. Charlier, J.C.; Gonze, X.; Michenaud, J.P. Graphite interplanar bonding: Electronic delocalization and van der Waals interaction. EPL Europhys. Lett. 1994, 28, 403. [CrossRef]

9. Hernandez, Y.; Nicolosi, V.; Lotya, M.; Blighe, F.M.; Sun, Z.; De, S.; Boland, J.J. High-yield production of graphene by liquid-phase exfoliation of graphite. Nat. Nanotechnol. 2008, 3, 563. [CrossRef] 
10. Ciesielski, A.; Samorì, P. Graphene via sonication assisted liquid-phase exfoliation. Chem. Soc. Rev. 2014, 43, 381-398. [CrossRef]

11. Zhu, Y.; Murali, S.; Cai, W.; Li, X.; Suk, J.W.; Potts, J.R.; Ruoff, R.S. Graphene and graphene oxide: Synthesis, properties, and applications. Adv. Mater. 2010, 22, 3906-3924. [CrossRef] [PubMed]

12. Zhu, Y.; James, D.K.; Tour, J.M. New routes to graphene, graphene oxide and their related applications. Adv. Mater. 2012, 24, 4924-4955. [CrossRef] [PubMed]

13. Gilje, S.; Han, S.; Wang, M.; Wang, K.L.; Kaner, R.B. A chemical route to graphene for device applications. Nano Lett. 2007, 7, 3394-3398. [CrossRef]

14. Eftekhari, A.; Garcia, H. The necessity of structural irregularities for the chemical applications of graphene. Mater. Today Chem. 2017, 4, 1-16. [CrossRef]

15. Tubón Usca, G.; Vacacela Gomez, C.; Fiallos, D.C.; Tavolaro, P.; Martino, G.; Caputi, L.S.; Tavolaro, A. Preparation of graphene oxide as biomaterials for drug adsorption. In AIP Conference Proceedings, College Park, MD, USA, 19 February 2015; American Institute of Physics: College Park, MD, USA, 2015; Volume 1646, pp. 79-86.

16. Fiallos, D.C.; Vacacela Gomez, C.; Tubon Usca, G.; Pérez, D.C.; Tavolaro, P.; Martino, G.; Tavolaro, A. Removal of acridine orange from water by graphene oxide. In AIP Conference Proceedings, College Park, MD, USA, 19 February 2015; American Institute of Physics: College Park, MD, USA, 2015; Volume 1646, pp. $38-45$.

17. Paton, K.R.; Varrla, E.; Backes, C.; Smith, R.J.; Khan, U.; O'Neill, A.; Higgins, T. Scalable production of large quantities of defect-free few-layer graphene by shear exfoliation in liquids. Nat. Mater. 2014, 13, 624. [CrossRef]

18. Vacacela Gomez, C.; Tene, T.; Guevara, M.; Tubon Usca, G.; Colcha, D.; Brito, H.; Molina, R.; Bellucci, S.; Tavolaro, A. Preparation of Few-Layer Graphene Dispersions from Hydrothermally Expanded Graphite. Appl. Sci. 2019, 9, 2539. [CrossRef]

19. Tubon Usca, G.; Vacacela Gomez, C.; Guevara, M.; Tene, T.; Hernandez, J.; Molina, R.; Caputi, L.S. Zeolite-Assisted Shear Exfoliation of Graphite into Few-Layer Graphene. Crystals 2019, 9, 377. [CrossRef]

20. He, T.; Dai, Q.; Huang, W.; Wang, X. Colloidal suspension of graphene oxide in ionic liquid as lubricant. Appl. Phys. A 2018, 124, 777. [CrossRef]

21. Wu, T.T.; Ting, J.M. Preparation and characteristics of graphene oxide and its thin films. Surf. Coat. Technol. 2013, 231, 487-491. [CrossRef]

22. Yan, J.A.; Chou, M.Y. Oxidation functional groups on graphene: Structural and electronic properties. Phys. Rev. B 2010, 82, 125403. [CrossRef]

23. Vacacela Gomez, C.; Robalino, E.; Haro, D.; Tene, T.; Escudero, P.; Haro, A.; Orbe, J. Structural and electronic properties of graphene oxide for different degree of oxidation. Mater. Today Proc. 2016, 3, 796-802. [CrossRef]

24. Savazzi, F.; Risplendi, F.; Mallia, G.; Harrison, N.M.; Cicero, G. Unravelling some of the structure-property relationships in graphene oxide at low degree of oxidation. J. Phys. Chem. Lett. 2018, 9, 1746-1749. [CrossRef]

25. Sheng, Y.; Tang, X.; Peng, E.; Xue, J. Graphene oxide based fluorescent nanocomposites for cellular imaging. J. Mater. Chem. B 2013, 1, 512-521. [CrossRef]

26. Lee, D.W.; Seo, J.W. sp2/sp3 carbon ratio in graphite oxide with different preparation times. J. Phys. Chem. C 2011, 115, 2705-2708. [CrossRef]

27. Talyzin, A.V.; Mercier, G.; Klechikov, A.; Hedenström, M.; Johnels, D.; Wei, D.; Moons, E. Brodie vs Hummers graphite oxides for preparation of multi-layered materials. Carbon 2017, 115, 430-440. [CrossRef]

28. Poh, H.L.; Šaněk, F.; Ambrosi, A.; Zhao, G.; Sofer, Z.; Pumera, M. Graphenes prepared by Staudenmaier, Hofmann and Hummers methods with consequent thermal exfoliation exhibit very different electrochemical properties. Nanoscale 2012, 4, 3515-3522. [CrossRef]

29. William, S.; Hummers, J.R.; Offeman, R.E. Preparation of graphitic oxide. J. Am. Chem. Soc. 1958, 80, 1339.

30. Marcano, D.C.; Kosynkin, D.V.; Berlin, J.M.; Sinitskii, A.; Sun, Z.; Slesarev, A.; Tour, J.M. Improved synthesis of graphene oxide. ACS Nano 2010, 4, 4806-4814. [CrossRef]

31. Chen, J.; Yao, B.; Li, C.; Shi, G. An improved Hummers method for eco-friendly synthesis of graphene oxide. Carbon 2013, 64, 225-229. [CrossRef]

32. Paredes, J.I.; Villar-Rodil, S.; Fernández-Merino, M.J.; Guardia, L.; Martínez-Alonso, A.; Tascon, J.M.D. Environmentally friendly approaches toward the mass production of processable graphene from graphite oxide. J. Mater. Chem. 2011, 21, 298-306. [CrossRef] 
33. Abdolhosseinzadeh, S.; Asgharzadeh, H.; Kim, H.S. Fast and fully-scalable synthesis of reduced graphene oxide. Sci. Rep. 2015, 5, 10160. [CrossRef] [PubMed]

34. Arao, Y.; Kubouchi, M. High-rate production of few-layer graphene by high-power probe sonication. Carbon 2015, 95, 802-808. [CrossRef]

35. Garino, N.; Castellino, M.; Sacco, A.; Risplendi, F.; Muñoz-Tabares, J.A.; Armandi, M.; Cicero, G. Proving the existence of Mn porphyrin-like complexes hosted in reduced graphene oxide with outstanding performance as oxygen reduction reaction catalysts. 2D Mater. 2019, 6, 045001. [CrossRef]

36. Randviir, E.P.; Brownson, D.A.; Banks, C.E. A decade of graphene research: Production, applications and outlook. Mater. Today 2014, 17, 426-432. [CrossRef]

37. Parvez, K.; Worsley, R.; Alieva, A.; Felten, A.; Casiraghi, C. Water-based and inkjet printable inks made by electrochemically exfoliated graphene. Carbon 2019, 149, 213-221. [CrossRef]

38. Ortega-Amaya, R.; Matsumoto, Y.; Flores-Conde, A.; Pérez-Guzmán, M.A.; Ortega-López, M. In situ formation of rGO quantum dots during GO reduction via interaction with citric acid in aqueous medium. Mater. Res. Express 2016, 3, 105601. [CrossRef]

39. Krishna, R.; Fernandes, D.M.; Venkataramana, E.; Dias, C.; Ventura, J.; Freire, C.; Titus, E. Improved reduction of graphene oxide. Mater. Today Proc. 2015, 2, 423-430. [CrossRef]

40. Ahirwar, S.; Mallick, S.; Bahadur, D. Electrochemical method to prepare graphene quantum dots and graphene oxide quantum dots. ACS Omega 2017, 2, 8343-8353. [CrossRef]

41. Xu, Y.; Bai, H.; Lu, G.; Li, C.; Shi, G. Flexible graphene films via the filtration of water-soluble noncovalent functionalized graphene sheets. J. Am. Chem. Soc. 2008, 130, 5856-5857. [CrossRef]

42. Bouchareb, S.; Doufnoune, R.; Riahi, F.; Cherif-Silini, H.; Lassaad, B. Non-covalent functionalization of graphene oxide using self-assembly of silver-triphenylphosphine for bactericidal formulations. Mater. Chem. Phys. 2019, 243, 122598. [CrossRef]

43. Eda, G.; Ball, J.; Mattevi, C.; Acik, M.; Artiglia, L.; Granozzi, G.; Chhowalla, M. Partially oxidized graphene as a precursor to graphene. J. Mater. Chem. 2011,21, 11217-11223. [CrossRef]

44. Babu, K.J.; Nahm, K.S.; Hwang, Y.J. A facile one-pot green synthesis of reduced graphene oxide and its composites for non-enzymatic hydrogen peroxide sensor applications. RSC Adv. 2014, 4, 7944-7951.

45. McAllister, M.J.; Li, J.L.; Adamson, D.H.; Schniepp, H.C.; Abdala, A.A.; Liu, J.; Aksay, I.A. Single sheet functionalized graphene by oxidation and thermal expansion of graphite. Chem. Mater. 2007, 19, 4396-4404. [CrossRef]

46. Bajpai, R.; Roy, S.; Rafiee, J.; Koratkar, N.; Misra, D.S. Graphene supported nickel nanoparticle as a viable replacement for platinum in dye sensitized solar cells. Nanoscale 2012, 4, 926-930. [CrossRef] [PubMed]

47. Jaworski, S.; Wierzbicki, M.; Sawosz, E.; Jung, A.; Gielerak, G.; Biernat, J.; Stobiński, L. Graphene oxide-based nanocomposites decorated with silver nanoparticles as an antibacterial agent. Nanoscale Res. Lett. 2018, 13, 116. [CrossRef] [PubMed]

48. Yin, F.; Wu, S.; Wang, Y.; Wu, L.; Yuan, P.; Wang, X. Self-assembly of mildly reduced graphene oxide monolayer for enhanced Raman scattering. J. Solid State Chem. 2016, 237, 57-63. [CrossRef]

49. Perumbilavil, S.; Sankar, P.; Priya Rose, T.; Philip, R. White light Z-scan measurements of ultrafast optical nonlinearity in reduced graphene oxide nanosheets in the 400-700 nm region. Appl. Phys. Lett. 2015, 107, 051104. [CrossRef]

50. Zamora-Ledezma, C.; Puech, N.; Zakri, C.; Grelet, E.; Moulton, S.E.; Wallace, G.G.; Poulin, P. Liquid crystallinity and dimensions of surfactant-stabilized sheets of reduced graphene oxide. J. Phys. Chem. Lett. 2012, 3, 2425-2430. [CrossRef]

51. Walch, N.J.; Nabok, A.; Davis, F.; Higson, S.P. Characterisation of thin films of graphene-surfactant composites produced through a novel semi-automated method. Beilstein J. Nanotechnol. 2016, 7, 209-219. [CrossRef]

(C) 2020 by the authors. Licensee MDPI, Basel, Switzerland. This article is an open access article distributed under the terms and conditions of the Creative Commons Attribution (CC BY) license (http://creativecommons.org/licenses/by/4.0/). 